\title{
Serious Game for Cognitive Testing of Elderly
}

\author{
Sangwoo Byun and Changhoon Park \\ Dept. of Game Engineering, Hoseo University, \\ 165 Sechul-ri, Baebang-myun, Asan, Chungnam 336-795, Korea \\ karan99@imrlab.hoseo.edu, chpark@hoseo.edu
}

\begin{abstract}
South Korea now has one of the lowest birth-rates in the world and one of the most rapidly aging populations. In such an aging society, the most representative disease threatening the quality of old person's life is 'Alzheimer's disease'. In this paper, we will propose a screening test based on game technology. And, this game will be designed by applying Fitt's law and Hick's law to test cognitive function.
\end{abstract}

Keywords: Screening tool, Accessibility, Cognitive function, Alzheimer's disease, Fitt's law, Hick's law.

\section{Introduction}

South Korea now has one of the lowest birth-rates in the world and one of the most rapidly aging populations. At this pace, Korean society is expected to become a socalled "aged society" by 2018, only 13 years after its designation as an "aging society" in 2000. In an aging society, people aged 65 or older make up over 7 percent of the population, while in an aged society this number exceeds 14 percent. It took France 115 years and the U.S. 72 years to go from an aging society to an aged society; Japan took 24 years for this change. In such an aging society, the most representative disease threatening the quality of old person's life is 'Alzheimer's disease(AD)', and this Alzheimer's disease is social and ethical problem beyond medical problem and it's an important problem waiting solution to improve people's welfare.

As the prevalence of dementia increases, and novel and better interventions to delay the progression of dementia become available, the detection of early dementia, especially in the primary care setting, becomes important. But, most of the present dementia screening test tools have difficulty detecting early dementia and are not easy used in the primary care setting. To overcome this problem, [1] proposes a Computerized Dementia Screening Test (CDST) consisting of four test items which are all computerized as one program: the block test for spatial span, the memory impairment screen of Buschke, the judgment of the line orientation test and the Go No-go test. In this paper, we will propose a screening test based on game technology to provide the fun factor to improve the accessibility of the test for a long time.

\section{Mild Cognitive Impairment (MCI)}

Mild cognitive impairment (MCI) is a syndrome defined as cognitive decline greater than expected for an individual's age and education level but that does not interfere notably 
with activities of daily life. MCI can thus be regarded as a risk state for dementia, and its identification could lead to secondary prevention by controlling risk factors such as systolic hypertension. Researchers have demonstrated the importance of identifying the declines in cognitive function indicative of imminent functional impairment. Therefore, investigators have been interested in the MCI prior to conversion to $\mathrm{AD}$ in recent era. Unfortunately, most of the present dementia screening test tools have difficulty detecting MCI and are not easy used in the primary care setting[2].

The diversity of the dementias and the diversity of the neuropsychological symptoms of dementia means that no single test is sufficient for the purpose of diagnosis or screening. Rather, a battery of neurocognitive tests is necessary. The commonality shared by all forms of dementia, however, indicates that an appropriate battery can comprise only a few well-chosen tests. This is important, because the major issue in dementia diagnosis is not necessarily to subtype the various forms of the condition, but rather to make the diagnosis as early as possible.

\section{Screening for MCI}

Computerized neurocognitive testing has been used in research since the days of the Commodore and the Apple 2e. The technology is well established in military and aerospace medicine, industrial medicine. Clinically, computerized tests are used routinely for attention deficit disorder (ADD) diagnosis and in sports medicine for concussion management. Computerized tests are reliable-in some respects more reliable than paper-and-pencil tests. They correlate well with conventional tests, are well-accepted by patients, and are capable of a high degree of accuracy. Some computerized tests generate results with millisecond accuracy, which is necessary for precise assessment of mild impairment in reaction times and information processing speed[3].

In this study, we propose a serious game for screening people with cognitive impairment. The fun factor of game can provide a way of monitoring humancomputer interaction naturally. Monitoring data from occurring gameplay will be used to detect sustained changes in person's cognitive performance.

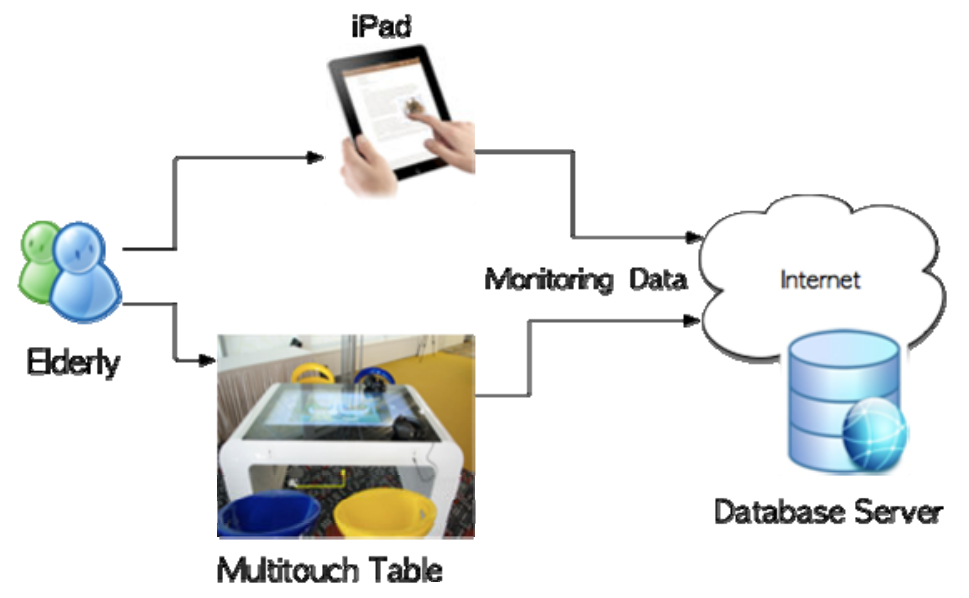

Fig. 1. Overview of system design 
We present three mini-games for reaction time, short-term memory and discernment respectively. For inferring cognitive performance, these games are designed based on Fitt's law and Hick's law. Fit's law is a model of human movement in human-computer interaction and ergonomics which predicts that the time required to rapidly move to a target area is a function of the distance to and the size of the target. And Hick's law describes describes the time it takes for a person to make a decision as a result of the possible choices he or she has. By means of these two laws, we will control the difficulty of the game in order to test cognitive function.

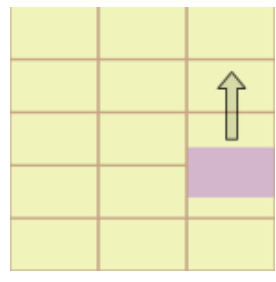

(1) Reaction time

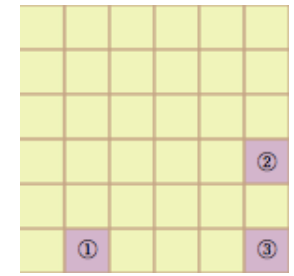

(2) Short-term memory

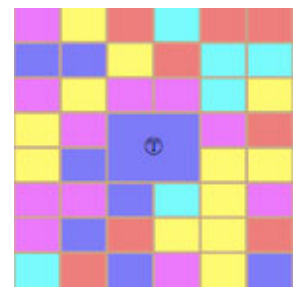

(3) Discernment

Fig. 2. Three different tests for cognitive function

In the reaction game, the user should touch a colored block that is moving in one direction starting from the outline. The game for short-term memory changes the color of tiles randomly and disappear. Then, the user should touch these tiles in the same order. And, the game for discernment changes the color of the center. The user should touch the same colored tile. The difficulty of these game is controlled by means of not only time, speed, or number of changing tiles but also the size of tile. So, we can obtain user interaction data during gameplay naturally.

The goal of this research is to develop unobtrusive techniques of collecting longterm data that will allow us to detect sustained trends in cognitive performance. The inferences will be used to classify significant performance changes, and additionally, to adapt computer interfaces with tailored hints and assistance when needed.

\section{Conclusion}

In this study, we proposed a serious game for screening people with cognitive impairment. The fun factor of game can provide a way of monitoring humancomputer interaction naturally. This game consisting of three test items: reaction time, short-term memory and discernment. Monitoring data from occurring gameplay will be used to detect sustained changes in person's cognitive performance. 


\section{References}

1. Cho, B., Yang, J., Kim, S., Yang, D.W., Park, M., Chey, J.: The validity and reliability of a Computerized Dementia Screening Test developed in Korea. Journal of the Neurological Sciences 203, 109-114 (2002)

2. Gualtieri, C.T.: Dementia Screening Using Computerized Tests. Journal of Insurance Medicine 36(3), 213-227 (2004)

3. Fillit, H.M., Simon, E.S., Doniger, G.M., Cummings, J.L.: Practicality of a computerized system for cognitive assessment in the elderly. Alzheimer's \& Dementia 4(1), 14-21 (2008) 